\title{
$24 E^{\mathrm{X}-641-67-297} E N i$ \\ 2 AA NASA-TM-X-55 8/73AE
}

\section{SPIN MAGNETIC EFFECT ON THE POLARIZATION OF THE ELECTRON-ATOM IMPACT RADIATIONG}

\section{BY \\ 6 LUE-Yung CHOW CHIU 9}

$N 67-29993$

CFSTI PRICE(S)

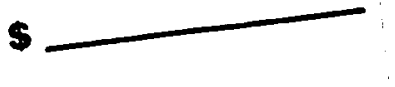

Hard copy (HC) $\frac{2.92}{165}$
Microfiche (MF) H 653 July 65

\section{NASA}

INAPA GODDARD SPACE FLIGHT CENTER GREENBELT, MARYLAND 3

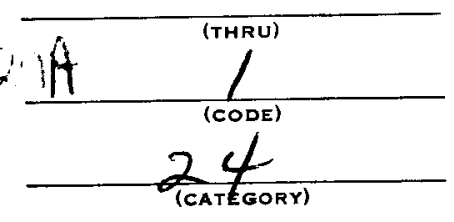

9 JUNE 196710 


\title{
SPIN MAGNETIC EFFECT ON THE POLARIZATION \\ OF THE ELECTRON-ATOM IMPACT RADIATION
}

\author{
by \\ Lue-Yung Chow Chiu* \\ Laboratory for Theoretical Studies, \\ National Aeronautics and Space Administration
}

Goddard Space Flight Center

Greenbelt, Maryland 


\title{
SPIN MAGNETIC EFFECT ON THE POLARIZATION \\ OF THE ELECTRON-ATOM IMPACT RADIATION
}

\author{
by
}

\section{Lue-Yung Chow Chiu*}

Laboratory for Theoretical Studies, National Aeronautics and Space Administration

*National Academy of Sciences - National Research Council Resident Research Associate

Goddard Space Flight Center

Greenbelt, Maryland 


\begin{abstract}
A new mechanism is proposed to explain the discrepancies between the measured and the expected polarization of the electron-atom impact radiation at threshold energy. The idea is the following: At threshold scattering, the spin of the scattered electron interacts magnetically with the orbital motion of the atomic electrons. The component of this magnetic interaction along the quantization axis, which is perpendicular to the incident electron direction, will split the magnetic sublevels of the atomic excited stated. This splitting will cause the coherent interference of the radiation, which originates from two degenerate upper levels and ends on a single lower level, to become ineffective. The polarization of the radiation is thereby affected and in fact decreases. The expected polarization $P$ is modified by a depolarization factor $f=\left(1+\overline{\omega^{2}} \tau^{2}\right)$, where $\omega$ is the frequency splitting of the excited state and $\tau$ is the life time of the transition from the excited upper state to a lower state. By using simple product wave function for the exceited state, the $\omega$ and hence the $P$ for various singlet-singlet transitions and triplet-triplet transitions have been calculated for helium atom. In general when the principal quantum number $\mathrm{n}$ of the excited state, where the radiation originates, increases, $f$ increases and approaches unity. Consequently $P$ increases and approaches the expected value. For example, $\mathrm{P}$ for $3{ }^{1} \mathrm{P} \rightarrow 2{ }^{1} \mathrm{~S}(5016 \mathrm{~A})$ line turns out to be $1.2 \%$ whereas for $4{ }^{1} \mathrm{D} \rightarrow 2{ }^{1} \mathrm{P}$ (4922A) line to be $50 \%$, and the expected values are $100 \%$ and $60 \%$ respectively.
\end{abstract}




\section{INTRODUCTION}

There has been increasing interest in the study of the polarization of light resulting from the excitation of the atom by the electron impact at threshold energy. This interest is specially centered on explaining the discrepancy between the theory $^{1,2}$ and the experimental ${ }^{3-6}$ results. When a helium atom at ground $1^{1} \mathrm{~S}$ state is excited to $\mathrm{n}^{1} \mathrm{P}$ state by electron impact at threshold energy, the outgoing electron having lost its linear momentum and hence its angular momentum can only be a S-wave. Due to conservation of the total angular momentum along the incident electron direction ( $z^{\prime}$-axis), only the magnetic sublevel $m_{\ell}=0$ of the ${ }^{1}{ }^{1} \mathrm{P}$ state can be excited (see Fig. 1). Here $\mathrm{m}_{\ell}$ is the component of the electronic angular momentum $\ell$ along the $z^{\prime}$-axis. The excited atom can then decay to the ground state by emitting $\pi$-radiation $\left(\Delta \mathrm{m}_{\ell}=0\right.$ transition). The radiation is therefore $100 \%$ polarized along the $z^{\prime}$-axis. If the atom is excited into $\mathrm{n}^{1} \mathrm{D}$ state and then decays into lower $\mathrm{n}^{\prime}{ }^{1} \mathrm{P}$ state, the radiation will

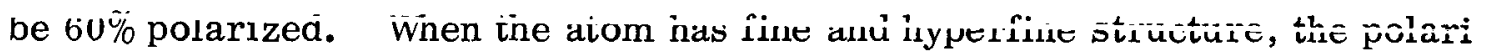
zation will be further decreased.

Earlier experimental results ${ }^{7.8}$ indicated that the threshold polarization was zero but rose to a maximum within a fraction of a volt. Recent measurements $^{3-5}$ on helium with improved techniques showed that except for $\lambda=4922 \mathrm{~A}$ $\left(4^{1} \mathrm{D} \rightarrow 2{ }^{1} \mathrm{P}\right)$ and $\lambda=4388 \mathrm{~A}\left(5^{1} \mathrm{D} \rightarrow 2^{1} \mathrm{P}\right)$ lines, the observed polarization in the 
immediate vicinity of threshold is still much too low to be comparable with the theoretical value. The most recent results of McFarland ${ }^{6}$ on line $\lambda=4922 \mathrm{~A}$ shows that the dipping of the polarization to a near threshold minimum of approximately $30 \%$ (expected value is $60 \%$ ) is a real effect. The general indication from the experimental observations is that when radiation originates from an upper state of high $n$ (principal quantum number), e.g. $5^{1} \mathrm{D}$, the threshold polarization is comparable to the theoretical value; whereas for low $\mathrm{n}$ states, the polarization remains very low.

In the present work, a new theory is developed to explain the discrepancies between the expected polarizations based on the simple conservation of angular momentum and the experimental results. At threshold scattering, the scattered electron having lost its momentum remains virtually stationary and very close to the excited atom such that its spin can interact magnetically with the orbital motion of the atomic electrons. This interaction is rather similar to the spinother-orbit interaction in an atomic system. The component of this magnetic interaction along the quantization axis (which is perpendicular to the incoming electron beam direction) will remove the degeneracy among the magnetic sublevels of the excited state and cause level splittings. The splittings will then affect the interference part of the resonance radiation and hence the polarization of the radiation. In fact the polarization $\mathrm{P}$, for the case without fine- and hyperfine-structure, will be shown (in Section 2) to be euqal to $P_{0}$, which is the polarization from conservation of angular momentum, multiplied by a 
depolarization factor $f=\left(1+\overline{\omega^{2}} \tau^{2}\right)^{-1}$. The $\overline{\omega^{2}}$ here is the square of the frequency separation between two interfering magnetic sublevels $\nu$ and $\nu \pm 2$ averaged over the spin orientations of the scattered electron, and $\tau$ is the life time for the excited state to decay to the lower state considered here. For high $n$ state of the excited helium, $\overline{\omega^{2}}$ is approximately proportional to $[2 n(2 n-1)$ $\times(2 n-2)]^{-2}$. Since $\tau^{\prime}$ s are roughly of the same order of magnitude, the depolarization factor $\mathrm{f} \rightarrow 1$ where $\mathrm{n}$ becomes large, say $\mathrm{n} \geq 5$, and consequently $\mathrm{P} \rightarrow \mathrm{P}_{0}$. For states of lower $n$, e. g. $n=2$ or $3, \overline{\omega^{2}} \tau^{2}$ is considerably larger than 1 so that both $f$ and $P$ are very small. The above conclusion seems to agree with the general experimental observations.

When the energy of the bombarding electron is slightly above the threshold, i.e., a fraction of electron volt, the selection rule $\Delta \mathrm{m}_{\ell}=0$ still holds to a good approximation, and $\mathrm{P}_{0}$ remains quite close to its threshold value. However the scattered electron may have gained enough linear momentum to get away from the atom and hence to make the spin-magnetic interaction and the frequency

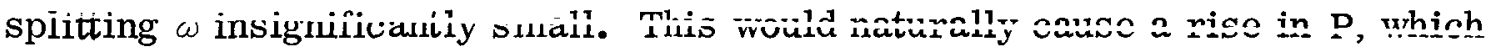
will then decrease as $\mathrm{P}_{0}$ decreases when the bombarding energy is increased further beyond its threshold value. This rise in $\mathrm{P}$ would only occur for cases where $\mathrm{P}$ is considerably smaller than $\mathrm{P}_{0}$ at threshold energy.

The depolarization factor considered above is similar to that derived by Breit $^{9}$ and later by Franken ${ }^{10}$ in their treatment of the polarization of the resonance fluorescence. There, the depolarization is due to a transverse 
magnetic field which is perpendicular to the polarization vector of the incident light. This effect was first observed ${ }^{11}$ at the crossing point of two excited fine-structure magnetic sublevels at certain magnetic field. This level cross ing technique has later been applied to determine the zero-field level structure. ${ }^{12}$ The depolarization effect at the zero-field crossing is also called Hanle effect ${ }^{13}$, which has been used to measure the excited state life times $^{12,14-17}$

The depolarization effect of the electron impact radiation at the threshol energy treated here is also magnetic in nature, and it can be looked upon as i due to an effective transverse magnetic field coming from the spin of the sca: tered electron. The general theory of this treatment will be developed in Section 2. The case with the fine- and the hyperfine-structure will be discus in Section 3. The numerical results on helium will be presented in the last Section. 


\section{GENERAL THEORY}

First we consider the simple case where the atom has no fine- and hyperfine-structure both in the ground and in the excited state. The atom is initially at the ground state $\underline{\mathrm{a}}, 1^{1} \mathrm{~S}_{0}$, and is being excited to state $\underline{\mathrm{b}}, \mathrm{n}{ }^{1} \mathrm{~L}_{\ell}$, by electron impact along the $z^{\prime}$-axis (shown in Fig. 1). The $z^{\prime}$-component of the total angular momentum of the initial system is therefore zero, i.e., $\mathrm{M}_{z^{\circ}}=0$. At threshold collision, the outgoing electron having lost its linear momentum, hence has zero angular momentum, becomes a spherical wave centered at the atomic nucleus (origin of the system). Due to the conservation of $\mathrm{M}_{z^{*}}$, atom can be excited to the magnetic sublevel $\phi_{\ell, 0}\left(\mathbf{r}^{\circ}\right)$ only. The subscript 0 means $\mathrm{m}_{\ell}=0$ and $\mathrm{m}_{\ell}$ is the $\mathrm{z}^{\prime}$-component of the atomic angular momentum $\ell$. Now we transform the coordinate system $\left(\mathbf{r}^{*}\right)$ into the system $(\mathbf{r})$ by rotating (clockwise) $\pi / 2$ around the $y^{\prime}$-axis (see Fig. 2). The wave function $\phi_{\ell, 0}\left(\mathbf{r}^{\prime}\right)$ is then transformed linearly into a set of wave functions $\phi_{\ell_{\nu}}(\mathbf{r})$ by the following:

$$
\begin{aligned}
\phi_{\ell, 0}\left(\mathbf{r}^{*}\right) & =\sum_{\nu} \mathrm{D}_{\nu, 0}^{\ell}(0, \pi / 2,0) \phi_{\ell_{\nu}}(\mathbf{r}) \\
& =[4 \pi /(2 \ell+1)]^{1 / 2} \sum_{\nu} \mathrm{Y}_{\ell_{\nu}}^{*}(\pi / 2,0) \phi_{\ell_{\nu}}(\mathbf{r})
\end{aligned}
$$

In this rotated ( $\mathbf{r}$ ) system, the incident electron is along the $\mathrm{x}$-axis. The subscript $\nu$ here is the projection of $\ell$ along the new $z$-azis, and the magnetic sublevels $\phi_{\ell_{\nu}}(\mathbf{r})$ with $\nu \neq 0$ as well as with $\nu=0$ can be populated. Including a 
time-dependent factor into (2.1), the time-dependent wave function of the excited state $\underline{b}$ is as follows:

$$
(4 \pi / 2 \ell+1)^{1 / 2} \sum_{\nu} \mathrm{Y}_{\ell_{\nu}}^{*}(\pi / 2,0) \phi \ell_{\nu}(\mathbf{r}) \exp \left(-\mathrm{i} \mathrm{E}_{\nu} / \hbar-\Gamma_{\mathrm{n} \ell} / 2\right) \mathrm{t}
$$

where $E_{\nu}$ is the energy of the sublevel $\nu \cdot \Gamma_{n} \ell$ is the decaying constant of state $\underline{b}$, and which is the reciprocal of the life time $\tau_{\mathrm{n} \ell}$, i.e., $\Gamma_{\mathrm{n} \ell}=1 / \tau_{\mathrm{n} \ell}$. The state $\underline{b}$ is then decaying via electric dipole transition into a lower state $\underline{c}\left(n^{\prime} L_{\ell^{\prime}}^{\prime}\right)$ which has decay constant $\Gamma_{n} \cdot \ell^{\prime}$ and a set of magnetic sublevels $\mu$. The instantaneous rate at which the radiation of polarization $\mathbf{q}$ is emitted during the transition process is

$$
\begin{gathered}
\mathrm{R}(\mathbf{q}, \mathrm{t})=\mathrm{A} \sum_{\mu} \mid\left\langle\sum_{\nu} \mathrm{Y}_{\ell_{\nu}}^{*}(\pi / 2,0) \phi_{\ell_{, \nu}}(\mathbf{r}) \mathrm{e}^{-\left(\mathrm{i} E_{\nu} / \hbar+\Gamma_{\mathrm{n}} \ell / 2\right) \mathrm{t}}\right. \\
\left.|\mathbf{q} \cdot \mathbf{r}| \phi_{\ell^{\prime}}(\mathbf{r}) \mathrm{e}^{-\left(\mathrm{i} E_{\mu} / \hbar+\Gamma_{\mathrm{n}} \cdot \ell^{\prime} / 2\right) \mathrm{t}}\right\rangle\left.\right|^{2}
\end{gathered}
$$

where the proportional constant A absorbs all the factors which are independent of the summation indices. Using the expression,

$$
\mathrm{q}_{\nu \mu}=\left\langle\phi_{\ell_{\nu}}(\mathbf{r})|\mathbf{q} \cdot \mathbf{r}| \phi_{\ell^{\prime} \mu}(\mathbf{r})\right\rangle
$$

for the electric dipole transition matrix, (2.3) now reads,

$$
\mathbf{R}(\mathbf{q}, \mathrm{t})=\mathrm{A} \sum_{\mu} \sum_{\nu} \sum_{\bar{\nu}} \mathrm{Y}_{\ell_{\nu}}(\pi / 2,0) \mathrm{Y}_{\ell \bar{\nu}}^{*}(\pi / 2,0) \mathrm{q}_{\nu \mu} \mathrm{q} \bar{\nu}_{\mu} \mathrm{e}^{\left(\mathrm{i} \omega_{\nu \bar{\nu}}-\Gamma_{\left.\ell \ell^{\prime}\right)} \mathrm{t}_{(2.5)}\right.}
$$


Where

$$
\omega_{\nu \bar{\nu}}=\left(E_{\nu}-E_{\bar{\nu}}\right) / \hbar \text { and } \Gamma_{\ell \ell}=\Gamma_{n} \ell+\Gamma_{n} \cdot \ell^{\prime} \cdot
$$

The indices $n, l, \nu, \bar{\nu}$ refer to the upper state $\underline{b}$ and $n^{\prime}, \ell^{\prime \prime}, \mu$ refer to the lower state c. Since the decay constant $\Gamma_{\ell \ell^{\prime \prime}}$ usually is large enough $\left(10^{7} \sim 10^{10} \mathrm{sec}^{-1}\right)$, that an atom is excited to state $\underline{b}$ and then decays to lower state $\underline{c}$ by emitting $a$ photon all in a time short compared with the time for this atom to be re-excited. We can then integrate the Eq. (2.5) over the time to obtain the rate $R(q)$,

$$
\begin{aligned}
R(\mathbf{q}) & =\int_{0}^{\infty} \mathrm{R}(\mathbf{q}, \mathrm{t}) \mathrm{d} \mathrm{t} \\
& =\mathrm{A} \tau \sum_{\mu} \sum_{\nu} \sum_{\bar{\nu}} \mathrm{Y}_{\ell_{\nu}}(\pi / 2,0) \mathrm{Y}_{\ell_{\bar{\nu}}}^{*}(\pi / 2,0) \mathrm{q}_{\nu \mu} \mathrm{q}_{\bar{\nu} \mu}^{*}\left(1-i \tau \omega_{\nu \bar{\nu}}\right)^{-1}
\end{aligned}
$$

where

$$
\tau=1 / \Gamma_{\ell \ell}
$$

is the life time of transition from state $\underline{b}$ to state $\underline{c} . R(q)$ will be nonvanishing only when

$$
\nu=\bar{\nu}, \bar{\nu} \pm 2
$$


Since

$$
\mathrm{x}_{\nu \mu} \mathrm{x}_{\bar{\nu} \mu}^{*}=\mathrm{y}_{\nu \mu} \mathrm{y}_{\bar{\nu} \mu}^{*} \text { when } \nu=\bar{\nu}
$$

and

$$
\mathrm{x}_{\nu \mu} \mathrm{x}_{\bar{\nu} \mu}^{*}=-\mathrm{y}_{\nu \mu} \mathrm{y}_{\bar{\nu} \mu}^{*} \text { when } \nu=\bar{\nu} \pm 2
$$

we can express $R(\mathbf{x})$ and $R(y)$ as follows:

$$
R(x)=R_{0}+R_{1}
$$

and

$$
R(y)=R_{0}-R_{1}
$$

where

and

$$
\mathbf{R}_{0}=\mathrm{A} \tau \sum_{\mu} \sum_{\nu}\left|\mathrm{Y}_{\ell_{\nu}}(\pi / 2,0)\right|^{2}\left|\mathrm{x}_{\nu \mu}\right|^{2}
$$

$$
\mathrm{R}_{1}=2 \mathrm{~A} \tau \sum_{\mu} \sum_{\nu>\bar{\nu}} \mathrm{Y}_{\ell_{\nu}}(\pi / 2,0) \mathrm{Y}_{\ell_{\bar{\nu}}}^{*}(\pi / 2,0) \mathrm{x}_{\nu \mu} \mathrm{x}_{\bar{\nu} \mu}^{*}\left(1+\tau^{2} \omega_{\nu \bar{\nu}}^{2}\right)^{-1}(2.12 \mathrm{a})
$$

The Zeeman level splitting $\hbar \omega_{\nu \bar{\nu}}=\mathrm{E}_{\nu}-\mathrm{E}_{\nu-2}$ is independent of the subscripts $\nu$ and $\bar{\nu}, \omega_{\nu \bar{\nu}}$ can therefore be replaced by $\omega$ and (2.12a) becomes

$$
\mathrm{R}_{1}=2 \mathrm{~A} \tau\left(1+\tau^{2} \omega^{2}\right)^{-1} \sum_{\mu} \sum_{\nu>\bar{\nu}} \mathrm{Y}_{\ell_{\nu}}(\pi / 2,0) \mathrm{Y}_{\ell_{\nu}}^{*}(\pi / 2,0) \mathrm{x}_{\nu \mu} \mathrm{x}_{\nu \mu}^{*}
$$

The polarization, $\mathrm{P}$, of the emitted radiation (observed along $\mathrm{z}$-axis) is as follows : 


$$
P=\frac{R(x)-R(y)}{R(x)+R(y)}=\frac{R_{1}}{R_{0}}=P_{0} f
$$

Where

$$
\mathrm{P}_{0}=\frac{2 \sum_{\mu} \sum_{\nu>_{\nu}} \mathrm{Y}_{\ell_{\nu}}(\pi / 2,0) \mathrm{Y}_{\ell_{\nu}}^{*}(\pi / 2,0) \mathrm{x}_{\nu \mu} \mathrm{x}_{\bar{\nu}}^{*}}{\sum_{\mu} \sum_{\nu}\left|\mathrm{Y}_{\ell_{\nu}}(\pi / 2,0)\right|^{2}\left|\mathrm{x}_{\nu, \mu}\right|^{2}}
$$

is the expected polarization by considering the conservation of angular momentum only, and

$$
f=\left(1+\tau^{2} \omega^{2}\right)^{-1}
$$

is the depolarization factor. When the state $\underline{b}$ is degenerate, i.e., $\omega=0, \mathrm{P}$ is equal to its maximum value $P_{0^{*}}$. It decreases through the factor $f$ when the Zeeman splitting (with respect to the new z-axis), $\omega$, becomes nonzero. If $\tau^{2} \omega^{2} \gg 1$, then $\mathrm{P} \rightarrow 0$, the emitted radiation is unpolarized. The above electric dipole matrix element $x_{\nu \mu}$ need not be evaluated. Since ${ }^{18}$

$$
r_{ \pm}=\mp(1 / \sqrt{ } 2)(x \pm i y) \text { or } x=-(1 / \sqrt{ } 2)\left(r_{ \pm}-r_{-1}\right)
$$

we have ${ }^{19}$

$$
x_{\nu \mu}=-(1 / \sqrt{ } 2)\left[\left(\ell^{\prime} 1 \ell ; \mu 1 \nu\right)-\left(\ell^{\prime} 1 \ell ; \mu,-1, \nu\right)\right]\left(\ell\|\mathbf{r}\| \ell^{\prime}\right) .
$$


The reduced matrix element $\left(\ell\|r\| \ell^{\prime}\right)$ will be cancelled in (2.14) and only Clebsch-Gordon coefficients $(\ell \cdot 1 \ell ; \mu, \pm 1, \nu)$ will remain in the expression for $P_{0}$ and $P$.

$R_{1}$ as shown in $(2.10 \mathrm{c})$, arising from two upper Zeeman levels $\nu$ and $\bar{\nu}$ decaying into a single lower level $\mu$, represents the interference part of the radiation, and which appears to be most effective when $\nu$ and $\bar{\nu}$ are degenerate (interfering coherently). But in the case of degeneracy, this interference cannot be regarded as true interference. The magnetic sublevels $\phi_{\ell, \nu}(\mathbf{r})$ in this case can always be combined linearly and transformed back into a single level $\phi_{\ell, 0}\left(\mathbf{r}^{\prime}\right)$ by rotating the coordinate system (r) back to $\left(\mathbf{r}^{\prime}\right)$. Naturally, no interference can arise when only a single upper level is populated. However, when fine- and hyperfine-structure exist (and when their magnetic sublevels are degenerate), the interference effect is a real effect. Because all the magnetic sublevels of the fine-structure, $\phi_{j_{, m_{j}}} \quad\left(\mathbf{r}^{\prime}\right)$, as well as that of the hyperfine-structure, $\phi_{\mathrm{f}_{\circ} \mathrm{m}_{\mathrm{f}}}\left(\mathbf{r}^{0}\right)$ can be populated (will be shown in the next section) in the initial $\left(\mathbf{r}^{\prime}\right)$ system.

Let us concentrate our problem again in this rotated (r) system, which is free from the external transverse magnetic field (which is parallel to the $\mathrm{z}$-axis and perpendicular to the incident electron beam to cause Zeeman splitting. A field which is parallel to the electron beam direction ( $\mathrm{x}$-axis) will obviously have no effect on the polarization $\mathrm{P}$, because it does not remove the degeneracy among the magnetic sublevels $\nu$ (which are quantized with respect to the $\mathrm{z}$-axis). The energy separation $\Delta \mathrm{E}_{\nu \bar{\nu}}=\hbar \omega_{\nu \bar{\nu}}$ here is considered to arise out of the magnetic 
interaction between the spin of the scattered electron and the orbital motion of the atomic electron. At threshold scattering, the outgoing electron having lost its momentum becomes a stationary spherical wave about the origin which is also the center of the atom. The excited atom is consisted of a shielded nuclear core and an outer electron at the atomic shell $(\mathrm{n} \ell)$. The scattered electron is electron 1 with coordinate $\mathbf{r}_{1}$ and spin $\mathbf{s}_{1}$. The excited atomic electron is electron 2 with coordinate $\mathbf{r}_{2}$, and its linear and angular momenta are $\mathbf{p}_{2}$ and $\ell_{2}(=\ell)$ respectively. The vector potential $\mathbf{A}$ at electron 2 due to the spin magnetic moment, $\mu_{s}$, of the electron 1 is,

$$
\mathbf{A}=\left(\mu_{\mathrm{s}} \times \mathbf{r}_{12}\right) / \mathbf{r}_{12}^{3}
$$

where $\mathbf{r}_{12}=\mathbf{r}_{1}-\mathbf{r}_{2}$. The interaction Hamiltonian of $\mathbf{p}_{2}$ under potential $\mathbf{A}$ is,

$$
\mathrm{H}=(\mathrm{e} / \mathrm{mc}) \mathbf{A} \cdot \mathbf{p}_{2}=(\mathrm{e} / \mathrm{mc})\left(\boldsymbol{\mu}_{\mathrm{s}} \cdot \mathbf{r}_{12} \times \mathbf{p}_{2}\right) / \mathrm{r}_{12}^{3}
$$

where $e$ and $\mathrm{m}$ are the electron charge and the electron mass respectively, and $c$ is the velocity of light. Since

$$
\mu_{s}=g_{s} \mu_{0} s_{1}=2 \mu_{0} s_{1},
$$

where $\mu_{0}=e \hbar / 2 \mathrm{mc}$ is the Bohr magneton, we express (2.19) in atomic units as follows: 


$$
\mathcal{H}=a^{2}\left(\mathbf{s}_{1} \cdot \mathbf{r}_{12} \times \mathbf{p}_{2}\right) / \mathbf{r}_{12}^{3}
$$

The fine structure constant $a=\mathrm{e}^{2 / \hbar c}$ is a dimensionless quantity. Equation (2.21) resembels the spin-other-orbit interaction between two electrons. The difference is that the interaction between $\mathbf{s}_{2}$ and $\mathbf{p}_{1}$ is not present here. Now we take the expectation value of over the product wave function of electron 1 and electron 2 where $s_{1 z}$ and $\ell_{2 z}$ are quantized with eigenvalues $\sigma$ and $\nu$ respectively.

$$
\mathcal{H}=\alpha^{2} \sigma\left\langle u\left(r_{1}\right) \phi\left(\mathbf{r}_{2}\right)\left|\left(\mathbf{r}_{12} \times \mathbf{p}_{2}\right)_{2} / r_{12}^{3}\right| u\left(r_{1}\right) \phi\left(\mathbf{r}_{2}\right)\right\rangle
$$

$u\left(r_{1}\right)$ and $\phi\left(r_{2}\right)$ are the electronic wave function of electron 1 and $2 . u\left(r_{1}\right)$ is a radial wave function only because the scattered electron is a $S$ wave (at threshold scattering).

Using the relation $\mathbf{p}=-i \nabla$, we express the operator in (2.22) as follows:

$$
\frac{1}{r_{12}^{3}}\left(\mathbf{r}_{12} \times \mathbf{p}_{2}\right)_{z}=\left(\frac{4 \pi}{3}\right)^{1 / 2}\left[\frac{Y_{1,-1}\left(\mathbf{r}_{12}\right)}{r_{12}^{2}} \nabla_{2}^{(1)}-\frac{Y_{1,1}\left(\mathbf{r}_{12}\right)}{r_{12}^{2}} \nabla(-1)\right]
$$

where

$$
\nabla_{2}^{( \pm 1)}=\mp \frac{1}{\sqrt{2}}\left(\frac{\partial}{\partial x_{2}} \pm i \frac{\partial}{\partial y_{2}}\right) \text { and } \nabla_{2}^{(0)}=\frac{\partial}{\partial z_{2}}
$$


are the spherical components of a gradient operator $Y_{1, \pm 1}\left(\mathbf{r}_{12}\right) / r_{12}^{2}$ is the irregular solid spherical harmonics of the first order and it can be expanded as follows: ${ }^{20,21}$

For $r_{2}>r_{1}$,

$$
\begin{gathered}
\frac{Y_{1}^{ \pm 1}\left(\mathbf{r}_{12}\right)}{r_{12}^{2}}=\sum_{\ell=1}^{\infty} \sum_{m=-l}^{\ell}(-)^{l}\left[\frac{4 \pi(2 \ell) !}{2(2 \ell-1) !}\right]^{1 / 2}(l-1, \ell, 1 ; \pm 1-m, m, \pm 1) \frac{r_{1}^{l-1}}{r_{2}^{l+1}} Y_{\ell-1, \pm 1-m}\left(\mathbf{r}_{1}\right) \\
\times Y_{\ell, m}\left(r_{2}\right)
\end{gathered}
$$

and for $r_{1}>r_{2}$

$$
\frac{\mathrm{Y}_{1}^{ \pm 1}\left(\mathbf{r}_{12}\right)}{\mathrm{r}_{12}^{2}}=\sum_{\ell=1}^{\infty} \sum_{\mathrm{m}=-\ell}^{\ell}(-)^{\ell+1}\left[\frac{4 \pi(2 \ell) !}{2(2 \ell-1) !}\right]^{1 / 2}(\ell-1, \ell, 1 ; \pm 1-\mathrm{m}, \mathrm{m}, \pm 1) \frac{\mathrm{r}_{2}^{\ell-1}}{\mathrm{r}_{1}^{\ell+1}} \mathrm{Y}_{\ell-1, \pm 1-\mathrm{m}}\left(\mathrm{r}_{2}\right)
$$

Now we use (2.25) to expand the operator in (2.23), which is then multiplied by $\left|\mathrm{u}\left(\mathrm{r}_{1}\right)\right|^{2}$ and integrated over the volume element $\mathrm{d} \mathbf{r}_{1}$ of electron 1 . Since $u\left(r_{1}\right)$ is angular independent, only the $l=1$ term in the expansion (2.25a) for case $r_{1}<r_{2}$ will contribute to the ingegral. We therefore have,

$$
\begin{aligned}
\int\left|\mathrm{u}\left(\mathrm{r}_{1}\right)\right|^{2} \frac{\left(\mathbf{r}_{12} \times \mathbf{p}_{2}\right)_{z}}{\mathrm{r}_{12}^{3}} \mathrm{~d} \mathbf{r}_{1} & =\left(\frac{4 \pi}{3}\right)^{1 / 2} \mathrm{f}\left(\mathrm{r}_{2}\right)\left[\frac{\mathrm{Y}_{1,-1}\left(\mathbf{r}_{2}\right)}{\mathrm{r}_{2}^{2}} \nabla{ }_{2}^{(1)}-\frac{\mathrm{Y}_{1,+1}\left(\mathbf{r}_{2}\right)}{\mathrm{r}_{2}^{2}} \nabla(-1)\right] \\
& =f\left(\mathrm{r}_{2}\right)\left(1 / \mathrm{r}_{2}^{3}\right)\left(\mathbf{r}_{2} \times \mathbf{p}_{2}\right)_{z}=f\left(\mathrm{r}_{2}\right)\left(1 / \mathrm{r}_{2}^{3}\right) \ell_{2 z}
\end{aligned}
$$

Where

$$
f\left(r_{2}\right)=-4 \pi \int_{0}^{r_{2}}\left|u\left(r_{1}\right)\right|^{2} r_{1}^{2} d r_{1}
$$


Using (2.26), the two electron integral,

$$
\begin{aligned}
I & \left.\left.=\left\langle\phi\left(\mathbf{r}_{2}\right) u\left(\mathbf{r}_{1}\right)\right|\left(\mathbf{r}_{12} \times \mathbf{p}_{2}\right)_{z} / \mathbf{r}_{12}^{3}\right) \phi\left(\mathbf{r}_{2}\right) u\left(r_{1}\right)\right\rangle \\
& =\left\langle\phi\left(\mathbf{r}_{2}\right)\left|\mathrm{f}\left(\mathbf{r}_{2}\right)\left(1 / \mathbf{r}_{2}^{3}\right) \ell_{2 z}\right| \phi\left(\mathbf{r}_{2}\right)\right\rangle=\nu Q
\end{aligned}
$$

becomes an one-electron integral. Where $\nu$ is the eigenvalue of $\ell_{2 z}$, and

$$
Q=\left\langle\phi\left(\mathbf{r}_{2}\right)\left|f\left(r_{2}\right)\left(1 / r_{2}^{3}\right)\right| \phi\left(\mathbf{r}_{2}\right)\right\rangle
$$

The expectation value over $\&$ now becomes,

$$
\langle\not 4\rangle=\alpha^{2} \sigma \nu Q
$$

The frequency separation (in atomic units) between sublevels $\nu$ and $\nu^{\circ}(=\nu-2)$ is,

$$
\omega=\Delta \mathrm{E}_{\nu, \nu-2}=2 \alpha^{2} \sigma \mathrm{Q}
$$

Since $\sigma$ is the eigenvalue of $s_{1 z}$, it can take the value of $+1 / 2$ or $-1 / 2$ depending on the orientation of $s_{1}$ with respect to the z-axis. However $\sigma^{2}(=1 / 4)$ is the same for both cases, which then stands for the average values, $\overline{\sigma^{2}}$, over the orientations. Using $\overline{\sigma^{2}}=1 / 4$, we have

$$
\overline{\omega^{2}} \tau^{2}=\alpha^{4} Q^{2} \tau^{2}
$$


and

$$
\mathrm{P}=\mathrm{P}_{0}\left(1+\alpha^{4} \mathrm{Q}^{2} \tau^{2}\right)^{-1}
$$

Now the remaining job is to evaluate $Q$ which is defined in (2.29). Q can be readily integrated when

$$
f\left(r_{2}\right)=-4 \pi \int_{0}^{r_{2}}\left|u\left(r_{1}\right)\right|^{2} r_{1}^{2} d r_{1}
$$

is known . Here $\left|u\left(r_{1}\right)\right|^{2}$, the density of the scattered electrion 1 , is fortunately needed only in the region $0 \leq r_{1} \leq r_{2}$. In this region, $u\left(r_{1}\right)$ is a $S$ wave outside a shielded nuclear core of +1 charge, and to a good approximation it can be written as,

$$
u\left(r_{1}\right)=(1 / \sqrt{\pi}) e^{-r_{1}}
$$

where $1 / \sqrt{ } \pi$ is the normalization constant. Substituting (2.34) into (2.27) we have

$$
f\left(r_{2}\right)=e^{-2 r_{2}}\left(2 r_{2}^{2}+2 r_{2}+1\right)-1
$$

The above equation of course would not be valid in region $r_{1}>r_{2}$. 


\section{EFFECT OF FINE- AND HYPERFINE-STRUCTURE}

Following the discussion in the last section, the atom which is initially in the ground state a with zero orbital angular momentum (S state) can only be excited to $\mathrm{m}_{\ell}=0$ sublevel of the excited state $\underline{\mathrm{b}}$ (which has principal quantum number $\underline{\mathrm{n}}$ and orbital angular momentum $\ell$ ) by election impact along the quantization z-axis at threshold energy. Now the state $\underline{b}$ has total electronic spin $s$, hence there exists fine structure interaction. The good quantum numbers in this case are $j$ and $m_{j}$, where $\mathbf{j}(=\ell+s)$ is the total electronic angular momentum and $m_{j}$ is the projection of $j$ along the $z^{\prime}$-axis. In $\left(j, m_{j}\right)$ representation, state $\underline{b}$ is as follows:

$$
\phi_{\mathrm{b}}\left(\mathbf{r}^{\prime}\right)=\sum_{\mathrm{m}_{\mathrm{s}}} \phi_{\ell, 0}\left(\mathbf{r}^{0}\right) \mathrm{x}_{\mathrm{s} \mathrm{m}_{\mathrm{s}}}=\sum_{\mathrm{jmj}}\left(\ell \mathrm{s} \mathrm{j} ; 0 \mathrm{~m}_{\mathrm{s}} \mathrm{m}_{\mathrm{j}}\right) \phi_{\mathrm{j} \mathrm{m} j}\left(\mathbf{r}^{\prime}\right)
$$

Where the Clebsch-Gordan coefficient $\left(\ell_{s} j ; 0 m_{s} m_{j}\right)$ is the weighing factor for each fine structure sublevel $\phi_{j \mathrm{~m}}, \mathrm{~m}_{\mathrm{s}}$ is the projection of $\mathrm{s}$ along the $\mathrm{z}^{\prime}$-axis, and the relation $\delta_{m_{s}, m_{j}}$ is implied. We again rotate (clockwise) the coordinate system $\left(\mathbf{r}^{\prime}\right) \pi / 2$ around the $y^{\prime}$-axis into the new system $(\mathbf{r})$, and $\phi_{j m j}\left(\mathbf{r}^{\prime}\right)$ is transformed into a linear combination of $\phi_{j \nu}(r)$. The subscript $\nu$ is the projection of $\mathbf{j}$ along the new z-axis. Equation (3.1) now becomes

$$
\phi_{\mathrm{b}}\left(\mathbf{r}^{\prime}\right)=\sum_{\mathbf{j} \nu} \mathrm{g}(\mathrm{j} \nu) \phi_{\mathrm{j} \nu}(\mathbf{r}),
$$


where 22

$$
g(j \nu)=\sum_{m_{s}}\left(\ell s j ; 0 m_{s} m_{s}\right) D_{\nu m_{s}}^{j}(0, \pi / 2,0) .
$$

The time-dependent wave function of state $\underline{b}$ is

$$
\phi_{\mathrm{b}}(\mathbf{r}, \mathbf{t})=\sum_{\mathrm{j} \nu} \mathrm{g}(\mathrm{j} \nu) \phi_{\mathrm{j} \nu}(\mathbf{r}) \mathrm{e}^{-\left(\mathrm{i} \mathrm{E}_{\mathrm{j} \nu} / \hbar+\Gamma_{\mathrm{r} \ell / 2) \mathrm{t}}\right.}
$$

and the rate of transition from state $\underline{b}$ to a lower state $\underline{c}$ (which has principal quantum number $\underline{\mathrm{n}}$, orbital angular momentum $\ell^{\prime}$ and fine structure sublevels $\phi_{j}{ }^{\prime}$ ) by emitting radiation of polarization $\mathbf{q}$ is (c.f. Eq. (2.6)),

$$
\begin{aligned}
\mathbf{R}(\mathbf{q})= & \mathrm{A} \tau \sum_{\mathbf{j} \overline{\mathbf{j}}} \sum_{\nu \bar{\nu}} \sum_{\mathbf{j}^{\prime} \mu} \mathrm{g}^{*}(\mathrm{j} \nu) \mathrm{g}(\overline{\mathrm{j}} \bar{\nu}) \mathrm{q}\left(\mathrm{j} \nu ; \mathrm{j}^{\prime} \mu\right) \mathrm{q}^{*}\left(\overline{\mathrm{j}} \bar{\nu} ; \mathrm{j}^{\prime} \mu\right) \\
& \times[1-\mathbf{i} \tau \omega(\mathrm{j} \nu ; \overline{\mathrm{j}} \bar{\nu})]^{-1}
\end{aligned}
$$

where

$$
\mathrm{q}\left(\mathrm{j} \nu ; \mathrm{j}^{\prime} \mu\right)=\left\langle\phi_{\mathrm{j} \nu}(\mathbf{r})|\mathbf{q} \cdot \mathbf{r}| \phi_{\mathbf{j}^{\prime} \mu}(\mathbf{r})\right\rangle
$$

and

$$
\omega(\mathrm{j} \nu ; \overline{\mathrm{j}} \bar{\nu})=\left(\mathrm{E}_{\mathrm{j} \nu}-\mathrm{E}_{\overline{\mathrm{j}} \bar{\nu}}\right) / \mathrm{h}
$$


The life time $\tau$ of transition between states $\ell$ and $\ell^{*}$ (which is defined in (2.7)) is independent of $j$ and $j^{\prime}$. A is a proportional constant which is independent of all the summation indices. Here the quantum numbers $l, j, \nu, \bar{j}, \bar{\nu}$ belong to the upper state $\underline{b}$ and $\ell^{\prime}, j^{\prime}, \mu$ belong to the lower state $\underline{c}$. We again split $R(q)$ into a linear combination of a interfering part $R_{1}$ and a non-interfering part $R_{0}$ such that,

$$
R(x)=R_{0}+R_{1} \text { and } R(y)=R_{0}-R_{1} \text {. }
$$

$R_{0}$ and $R_{1}$ have the following expressions:

where

$$
R_{0}=A \tau \sum_{j j^{\prime}} R_{0}\left(j j^{\prime}\right)
$$

$$
R_{0}\left(j j^{\prime}\right)=\sum_{\nu \mu}|g(j \nu)|^{2}\left|x\left(j \nu ; j^{\prime} \mu\right)\right|^{2},
$$

and

$$
\begin{aligned}
\mathbf{R}_{1}= & 2 \mathrm{~A} \tau \sum_{\mathbf{j}^{\prime} \mu} \sum_{\mathbf{j} \nu>\bar{j}_{\bar{\nu}}} \mathrm{g}^{*}(\mathbf{j} \nu) \mathrm{g}(\overline{\mathbf{j}} \bar{\nu}) \times\left(\mathbf{j} \nu ; \mathbf{j}^{\prime} \mu\right) \mathbf{x}^{*}\left(\overline{\mathbf{j}} \bar{\nu} ; \mathbf{j}^{\prime} \mu\right) \\
& \times\left[1+\tau^{2} \omega^{2}(\mathbf{j} \nu ; \bar{j} \bar{\nu})\right]^{-1}
\end{aligned}
$$

Since the fine structure separations are usually large enough that

$$
\tau^{2} \omega^{2}(\mathrm{j} \nu ; \overline{\mathrm{j}} \bar{\nu}) \gg 1 \text { for } \mathrm{j} \neq \overline{\mathrm{j}}
$$


we can neglect the terms of $j \neq \bar{j}$ in (3.11) and rewrite $R_{1}$ as follows:

$$
\mathrm{R}_{1} \approx \mathrm{A} \tau \sum_{\mathrm{j} \mathrm{j}^{\prime}} \mathrm{R}_{1}\left(\mathrm{j} \mathrm{j}^{\prime}\right)
$$

and

$$
\mathrm{R}_{1}\left(\mathrm{j} \mathrm{j}^{\prime}\right)=2\left(1+\tau^{2} \omega_{\mathrm{j}}^{2}\right)^{-1} \sum_{\mu} \sum_{\nu>\bar{\nu}} \mathrm{g}^{*}(\mathrm{j} \nu) \mathrm{g}(\mathrm{j} \bar{\nu}) \mathbf{x}\left(\mathrm{j} \nu ; \mathrm{j}^{\prime} \mu\right) \mathbf{x}^{*}\left(\mathrm{j} \bar{\nu} ; \mathrm{j}^{\prime} \mu\right)(3
$$

where

$$
\omega_{j}=\left(E_{j \nu}-E_{j \nu-2}\right) / \hbar
$$

is independent of $\nu$. The polarization of the radiation is therefore,

$$
P=\frac{R(x)-R(y)}{R(x)+R(y)}=\frac{R_{1}}{R_{0}}=\frac{\sum_{j j^{\prime}} R_{1}\left(j j^{\prime}\right)}{\sum_{j j^{\prime}} R_{0}\left(j j^{\prime}\right)}
$$

In the above expression for $\mathrm{P}$, we have included the radiation arising from all the allowed transitions between the fine structure levels $j$ of the upper state $\underline{b}$ and levels $j^{\prime}$ of the lower state $\underline{c}$. When each fine structure line, e.g. radiation due to a single upper level $j$ to a single lower level $j^{\prime}$ can be resolved, the polarization $P\left(\mathrm{j}^{\prime}\right)$ of each line is expressed as follows:

$$
P\left(j j^{\prime}\right)=\frac{R_{1}\left(j j^{\prime}\right)}{R_{0}\left(j j^{\prime}\right)}=P_{0}\left(j j^{\prime}\right) f_{j}
$$


where

$$
\mathbf{f}_{\mathbf{j}}=\left(1+\tau^{2} \omega_{\mathbf{j}}^{2}\right)^{-1},
$$

and

$$
P_{0}\left(j j^{\prime}\right)=2\left[R_{0}\left(j j^{\prime}\right)\right]^{-1} \sum_{\mu} \sum_{\nu>\bar{\nu}} g^{*}(j \nu) g(j \nu) x\left(j \nu ; j^{\prime} \mu\right) x^{*}\left(j \bar{\nu} ; j^{\prime} \mu\right)
$$

and $R_{0}\left(j j^{\prime}\right)$ is defined in (3.10).

When the nuclear spin of the atom is non-zero, $\mathbf{i} \neq 0$, hyperfine structure interaction exists. The excited state $\underline{b}$ is then a linear combination of eigenstates $\phi_{\mathrm{fm}}\left(\mathbf{r}^{\prime}\right)$, where $\mathbf{f}(=\mathbf{i}+\mathbf{j})$ is the total angular momentum of the atom and $m_{f}$ is the projection of $\mathbf{f}$ along the $z^{\prime}$-axis. After the coordinate system $\left(\mathbf{r}^{\prime}\right)$ has been rotated into the $(\mathbf{r})$ system by a rotation of $\pi / 2$ around the $y^{\prime}$-axis, we have

$$
\phi_{\mathrm{b}}\left(\mathbf{r}^{\prime}\right)=\sum_{\mathrm{j} f \nu} \mathrm{g}(\mathrm{j} \mathrm{f} \nu) \phi_{\mathrm{j} f \nu}(\mathbf{r})
$$

where

$$
g(j f \nu)=\sum_{m_{f}} \sum_{m_{s}}\left(j \text { if } ; m_{s} m_{i} m_{f}\right)\left(\ell s j ; 0 m_{s} m_{s}\right) \times D_{\nu m_{f}}^{f}(0, \pi / 2,0)
$$

$\nu$ here now is the projection of the $f$ along the new $z$-axis. The rate of radiation $R(q)$ is again split into a linear combination of $R_{0}$ and $R_{1}$, such that, 


$$
R(x)=R_{0}+R_{1} \text { and } R(y)=R_{0}-R_{1}
$$

and

$$
\text { polarization } P=R_{1} / R_{0}
$$

$R_{0}$ and $R_{1}$ are expressed as follows:

$$
R_{0}=A \tau \sum_{j j^{\prime}} \sum_{f f^{\prime}} \sum_{\nu \mu}|g(j f \nu)|^{2}\left|x\left(j f \nu ; j^{\prime} f^{\prime} \mu\right)\right|^{2}
$$

and

$$
\begin{gathered}
\mathrm{R}_{1}=\mathrm{A} \tau \sum_{\mathrm{j} \mathrm{j}^{\prime}} \sum_{\mathrm{f}^{\prime} \mu} \sum_{\mathrm{f} \nu>\mathrm{f}_{\bar{\nu}}} 2 \mathrm{~g}^{*}(\mathrm{j} \mathrm{f} \nu) \mathrm{g}(\mathrm{j} \overline{\mathrm{f}} \bar{\nu}) \times\left(\mathrm{j} f \nu ; \mathrm{j}^{\prime \prime} \mathrm{f}^{\prime} \mu\right) \mathrm{x}^{*}\left(\mathrm{j} \overline{\mathrm{f}} \bar{\nu} ; \mathrm{j}^{\prime} \mathrm{f}^{\prime} \mu\right) \\
(3.25) \\
\times\left[1+\tau^{2} \omega_{\mathrm{j}}^{2}(\mathrm{f} \nu ; \overline{\mathrm{f}} \bar{\nu})\right]^{-1}
\end{gathered}
$$

where

$$
\mathbf{x}\left(\mathrm{j} f \nu ; \mathrm{j}^{\prime} \mathrm{f}^{\prime} \mu\right)=\left\langle\phi_{\mathrm{jf} \nu}(\mathbf{r})|\mathbf{x}| \phi_{\mathrm{j}^{\prime} \mathrm{f}^{\prime}{ }_{\mu}}(\mathbf{r})\right\rangle
$$

and

$$
\omega_{j}(f \nu ; \bar{f} \bar{\nu})=\left(E_{j f \nu}-E_{j f} \nu^{\prime}\right) / \hbar
$$

In the above equations, the quantum numbers $j, f, \bar{f}, \nu, \bar{\nu}$ belong to the upper state $\underline{b}$ and the quantum numbers $\mathrm{j}^{\prime}:, \mathrm{f}^{\prime \prime}, \mu$ belong to the lower state $\underline{c}$. The life 
time $\tau$ is the same as defined in (2.7). In writing down the expression for $R_{1}$ in (3.25), we have assumed that the frequency splitting between the fine structure level $\mathrm{j}$ and the level $\overline{\mathrm{j}} \neq \mathrm{j}$ is large so that the terms of $\mathrm{j} \neq \overline{\mathrm{j}}$ have been neglected. If the hyperfine splitting is also large that

$$
\tau^{2} \omega_{\mathrm{j}}^{2}(\mathrm{f} \nu ; \overline{\mathrm{f}} \bar{\nu}) \gg 1 \text { for } \mathrm{f} \neq \overline{\mathrm{f}}
$$

then

$$
\begin{aligned}
R_{1}=A \tau \sum_{j f} 2\left(1+\tau^{2} \omega_{j f}^{2}\right) & {\left[\sum_{j^{\prime} f^{\prime} \mu} \sum_{\nu>\bar{\nu}} g^{*}(j f \nu) g(j f \bar{\nu}) \times\left(j f \nu ; j^{\prime} f^{\prime} \mu\right)\right.} \\
& \left.\times x^{*}\left(j f \bar{\nu} ; j^{\prime} f^{\prime} \mu\right)\right]
\end{aligned}
$$

where

$$
\omega_{j f}=\left(E_{j f \nu}-E_{j f \nu-2}\right) / \hbar .
$$

Similar to the dipole matrix element $x_{\nu \mu}$ in the last section (equation 2.17), the matrix element $x\left(j \nu ; j^{\prime} \mu\right)$ and $x\left(j f \nu ; j^{\prime} f^{\prime \prime} \mu\right)$ can also be expressed in terms of reduced matrix element $\left(\ell\|\mathbf{r}\| \ell^{\prime}\right)$ as follows: ${ }^{19}$

$$
\begin{aligned}
\mathbf{x}\left(\mathrm{j} \nu ; \mathrm{j}^{\prime} \mu\right)= & (1 / \sqrt{ } 2)(-)^{\mathrm{s}-\ell^{\prime}-\mathrm{j}}\left[\left(\mathrm{j}^{\prime} 1 \mathrm{j} ; \mu 1 \nu\right)-\left(\mathrm{j}^{\prime} 1 \mathrm{j} ; \mu,-1, \nu\right)\right] \\
& \times\left[(2 \ell+1)\left(2 \mathrm{j}^{\prime}+1\right)\right]^{1 / 2} \mathrm{~W}\left(\ell^{\prime} \mathrm{j}^{\prime} \ell \mathrm{j} ; \mathrm{s} 1\right)\left(\ell\|\mathbf{r}\| \ell^{\prime}\right)
\end{aligned}
$$


and

$. x\left(j f \nu ; j^{\prime} f^{\prime \prime} \mu\right)=-(1 / \sqrt{ } 2)(-)^{i+s-l^{\prime}-j^{\prime}-j-f}\left[\left(f^{\prime} 1 f ; \mu 1 \nu\right)-\left(f^{\prime} 1 f ; \mu,-1, \nu\right)\right]$

$-\times\left[(2 \ell+1)(2 j+1)\left(2 j^{\prime}+1\right)\left(2 f^{\prime}+1\right)\right]^{1 / 2} W\left(j^{\prime} f^{\prime} j f ; i 1\right) W\left(\ell^{\prime} j^{*} \ell j ; s 1\right)\left(\ell\|\mathbf{r}\| \ell^{\prime}\right)$

Where W (abcd; ef ) is the Racah coefficient ${ }^{23}, \ell$ and $\ell^{\prime}$ are the orbital angular momentum of the excited upper state $\underline{b}$ and the lower state $\underline{c}$ respectively. The reduced matrix element will be cancelled in the ratio in evaluating the polarization $\mathrm{P}$, and only Racah and Clebsch-Gordan coefficients will remain. 


\section{NUMERICAL VALUES FOR He ATOM}

A helium atom at the ground $1^{1} \mathrm{~S}_{0}$ state can be excited either into a singlet excited state $n^{1} L_{\ell}$ or a triplet excited state $n^{3} L_{j}$. In the case of a singlet state, we need to calculate the frequency splitting $\omega=2 a^{2} \sigma Q$ of Eq. (2.31). The integral Q which is defined in (2.29) has the following expression:

$$
\mathrm{Q}=\left\langle\phi_{\mathrm{n}} \ell\left(\mathbf{r}_{2}\right)\left|\mathrm{f}\left(\mathrm{r}_{2}\right)\left(1 / \mathrm{r}_{2}^{3}\right)\right| \phi_{\mathrm{n}} \ell\left(\mathbf{r}_{2}\right)\right\rangle .
$$

where $f\left(r_{2}\right)$ is shown in (2.35), and $\phi_{n} \ell \quad\left(r_{2}\right)$ is the wave function for the outer excited electron 2 of the helium. The inner (unexcited) electron, which remains at the $1 \mathrm{~s}$ orbital, does not contribute to the frequency splitting $\omega$. We use the following approximate wave function for $\phi_{\mathrm{n} \ell}\left(\mathbf{r}_{2}\right)$ :

$$
\phi_{n} \ell\left(\mathbf{r}_{2}\right)=(2 n !)^{-1 / 2}(2 \zeta)^{n+1 / 2} r_{2}^{n-1} e^{-\zeta r_{2}} Y_{\ell_{m}}\left(\theta_{2} \varphi_{2}\right)
$$

The $\zeta$ values are chosen by Slater's rules. ${ }^{24}$ We will calculate the polarization of the following transitions:

$$
\begin{gathered}
2^{1} \mathrm{P} \rightarrow 1^{1} \mathrm{~S}, \quad 3^{1} \mathrm{P} \rightarrow 2^{1} \mathrm{~S}, \quad 3^{1} \mathrm{D} \rightarrow 2^{1} \mathrm{P} \\
4^{1} \mathrm{D} \rightarrow 2^{1} \mathrm{P} \text { and } \quad 5^{1} \mathrm{D} \rightarrow 2^{1} \mathrm{P}
\end{gathered}
$$


The chosen $\zeta$ values for the corresponding upper state of the above transitions are, for $\mathrm{He}$,

$$
\zeta(2 \mathrm{p})=0.575, \zeta(3 \mathrm{p})=0.333, \zeta(3 \mathrm{~d})=0.333, \zeta(4 \mathrm{~d})=0.270 \text { and } \zeta(5 \mathrm{~d})=0.250
$$

Using the wave function in (4.2) and the expression in (2.35) for $f\left(r_{2}\right)$, integral $Q$ in (4.1) is integrated into the following:

$$
Q=-\frac{(2 \zeta)^{2 n+1}}{2 n(2 n-1)(2 n-2)}\left\{\frac{1}{(2 \zeta)^{2 n-2}}-\frac{2(2 n-1)(2 n-2)}{(2 \zeta+2)^{2 n}}-\frac{2(2 n-2)}{(2 \zeta+2)^{2 n-1}}-\frac{1}{(2 \zeta+2)^{2 n-2}}\right\}
$$

The values of $Q$ for all the above mentioned excited states are calculated and listed in Table I. The depolarization factor $\mathrm{f}$,

$$
f=\left(1+\overline{\omega^{2}} \tau^{2}\right)^{-1}=\left(1+\alpha^{4} Q^{2} \tau^{2}\right)^{-1}
$$

as well as the polarization $P$ from $(2.33)$,

$$
P=P_{0} f=P_{0}\left(1+\alpha^{4} Q^{2} \tau^{2}\right)^{-1}
$$

are thus obtained and listed in Table I. $\mathrm{P}_{0}$, which is the threshold polarization by conservation of angular momentum is calculated by (2.14) and is also listed in Table I. The lifetime $\tau$ used here are those calculated by Goldberg ${ }^{25}$. When the principal quantum number $n$ becomes large, say $n \geq 3$, the last three terms in the curly bracket of $(4.5)$ become negligibly small comparing to the first term. If we neglect these three terms Q becomes, 


$$
Q \approx-(2 \zeta)^{3} /[2 n(2 n-1)(2 n-2)] \text { when } n \geq 3
$$

The above approximate expression for $\mathrm{Q}$ indicates that $|\mathrm{Q}|$ decreases as $\mathrm{n}$ increases. Since the life time $\tau$ of the excited singlet states are of the same order of magnitude, the depolarization factor $f$ increases as $n$ increases (see Table I). When $n \geq 5$, $f \rightarrow 1$ and hence $P \rightarrow P_{0}$. In fact this general conclusion on the $n$-dependence of the polarization agrees very well with the experimental observations. ${ }^{3,4}$ Our calculation on individual transitions is by no means exact considering the approximations included, and it will be improved when better wave functions are used. Nevertheless this new mechanism with a simple minded calculation does seem to explain satisfactorily the discrepancies between the expected threshold polarizations, namely $\mathrm{P}_{0}$ 's here, and the observed values.

When helium atom is excited into a triplet state, $n^{3} \mathrm{~L}$, and then decays into a lower triplet state, $\mathrm{n}^{\prime}{ }^{3} \mathrm{~L}^{\prime}$, by electric dipole transition, the total polarization of the radiation shown in (3.16) cannot be written in a form similar to that in (4.7) for singlet transitions. However, when fine structure lines are resolved, the polarization between a pair of fine structure levels $\mathrm{j}$ (of the upper state) and $j^{\prime}$ (of the lower state) can be measured. This polarization, which is shown in $(3.17)$,

$$
P\left(j, j^{\prime}\right)=P_{0}\left(j j^{\prime}\right) f_{j}=\frac{P_{0}\left(j j^{\prime}\right)}{1+\tau^{2} \overline{\omega_{j}^{2}}}
$$


is quite similar to the expression in (4.7). Here we need to know $\overline{\omega_{j}^{2}}$. Instead of evaluating the integral I as shown in (2.28) for the singlet states, now we evaluate the following integral $I_{j}$ in the $(j \nu)$ representation,

$$
I_{j}=\left\langle\phi_{j \nu}\left(\mathbf{r}_{2}, \mathbf{r}_{3}\right)\left|f\left(r_{2}\right)\left(1 / r_{2}^{3}\right) \ell_{22}\right| \phi_{j \nu}\left(\mathbf{r}_{2}, \mathbf{r}_{3}\right)\right\rangle
$$

where $\mathbf{r}_{2}$ and $\mathbf{r}_{3}$ are the coordinates of the outer (excited) and the inner electrons respectively. Using Wigner-Eckart theorem,${ }^{18}(4.10)$ is expressed as a product of a reduced matrix element in $\ell$ representation and a coefficient which contains quantum numbers $\nu, j, l$ and $s$,

$$
\mathbf{I}_{\mathbf{j}}=\frac{\nu[\mathbf{j}(\mathbf{j}+1)+\ell(\ell+1)-s(\mathrm{~s}+1)]}{2 \mathbf{j}(\mathbf{j}+1)[\ell(\ell+1)]^{1 / 2}}\left(\ell\left\|f\left(\mathbf{r}_{2}\right)\left(1 / \mathbf{r}_{2}^{3}\right) \ell_{2}\right\| \ell\right)
$$

The orbital wave function $\phi_{n} \ell\left(\mathbf{r}_{2}, \mathbf{r}_{3}\right)$ of the excited helium atom is again approximated by a simple product wave function $\phi_{n} \ell\left(\mathbf{r}_{2}\right) \phi_{1 \mathrm{~s}}\left(\mathbf{r}_{3}\right)$, and the reduced matrix element becomes,

$$
\left(l\left\|f\left(r_{2}\right)\left(1 / r_{2}^{3}\right) l_{2}\right\| l\right)=[\ell(l+1)]^{1 / 2} Q
$$

where

$$
Q=\left\langle\phi_{n} \ell\left(\mathbf{r}_{2}\right)\left|f\left(r_{2}\right)\left(1 / r_{2}^{3}\right)\right| \phi_{n} \ell\left(r_{2}\right)\right\rangle
$$

is the same as that in (4.1) for singlet states. Following (2.22) the expectation value of $d f$ now is, 


$$
\langle\dot{d}\rangle=a^{2} \sigma I_{j}=\nu a^{2} \sigma[j(j+1)+l(l+1)-s(s+1)] Q /[2 j(j+1)] .
$$

Where $\sigma$ is the projection of electron spin of the scattered electron along the $z$-axis, and which can take values $+1 / 2$ or $-1 / 2$. The frequency separation (in atomic units) between $(\mathrm{j} \nu)$ and $(\mathrm{j}, \nu-2)$ becomes

$$
\omega_{\mathbf{j}}=\alpha^{2} \sigma Q \mathbf{G}_{\mathbf{j}}
$$

and

$$
\overline{\omega_{j}^{2}}=\alpha^{4} Q^{2} G_{j}^{2} / 4
$$

where

$$
G_{j}=[j(j+1)+\ell(l+1)-s(s+1)] / j(j+1) .
$$

Substituting (4.15) into (4.9) we have,

$$
P\left(j j^{\prime}\right)=P_{0}\left(j j^{\prime}\right) f_{j}
$$

and

$$
f_{j}=\left(1+\alpha^{4} Q^{2} G_{j}^{2} \tau^{2 / 4}\right)^{-1}
$$


When the fine structure lines are not resolved, the total polarization for $\mathrm{n}^{3} \mathrm{~L} \rightarrow$ $\mathrm{n}^{\prime}{ }^{3} \mathrm{~L}^{\prime}$ transition is (by (3.16)),

$$
P=\frac{\sum_{j j^{\prime}} f_{j} R_{1}^{0}\left(j j^{\prime}\right)}{\sum_{j j^{\prime}} R_{0}\left(j j^{\prime}\right)}
$$

where

$$
R_{1}^{0}\left(j^{\prime}{ }^{\prime}\right)=R_{1}\left(j j^{\prime}\right) f_{j}^{-1}
$$

and $R_{1}\left(j j^{\prime}\right)$ and $R_{0}\left(j j^{\prime}\right)$ are defined in (3.14) and (3.10) respectively. The $P_{0}$ in this case will be

$$
P_{0}=\lim _{\substack{f_{j} \rightarrow 1 \\ \text { foral1 } j}}=\frac{\sum_{j j^{\prime}} R_{1}^{0}\left(j j^{\prime}\right)}{\sum_{j j^{\prime}} R_{0}\left(j^{\prime}\right)},
$$

which has the same meaning as before, namely it is the threshold polarization when only the simple conservation law of angular momentum is considered.

In Table II we have listed the Q's, $\mathrm{P}_{0}$ 's and $\mathrm{P}^{\prime} \mathrm{s}$ for transitions $3^{3} \mathrm{P} \rightarrow 2^{3} \mathrm{~S}$, $4{ }^{3} \mathrm{D} \rightarrow 2{ }^{3} P$. The $P_{0}\left(j j^{\prime}\right), P\left(j j^{\prime}\right)$ and $\omega_{j}$ for fine structure lines have also been listed. The depolarization factors f's and consequently polarization $\mathrm{P}^{\prime} \mathrm{s}$ here for triplet transitions are considerably smaller than those of singlet transitions. This is due to the fact that the life time of triplet transitions are nearly two orders of magnitude longer than that of singlet transitions. 
In addition, the fine structure effect would also decrease the $\mathrm{P}_{0}{ }^{\prime} \mathrm{s}$ and hence the $\mathrm{P}^{\prime} \mathrm{s}$. The $\mathrm{P}_{0}{ }^{\prime} \mathrm{s}$ in Table $\mathrm{I}$ for singlet transitions agree with those previously calculated.1.2 However, the $\mathrm{P}_{0}$ 's in Table II for triplet transitions do not agree with all the previous values. For example the $P_{0}$ for $3^{3} \mathrm{P} \rightarrow 2{ }^{3} \mathrm{~S}$ transition was shown ${ }^{1.2}$ to be $36.6 \%$, whereas our value is $12 \%$. The reason is that we have included the interference effect in the present treatment while it has been neglected previously. For singlet transitions and for the case of complete degeneracy, this interference is not a true effect as has been discussed in Section 2. Since $\mathrm{P}_{0}$ corresponds to the value of $\mathrm{P}$ when magnetic sublevels are degenerate, i.e., $\omega=0$, it will be the same whether the interference effect has been included or not in computing $P_{0}$ for the singlet transitions. The situations of course will be different for the triplet transitions, because the interference remains effective even when the sublevels are degenerate, i.e., $\omega_{j}=0$

\section{ACKNOWLEDGMENT}

The author wishes to thank Dr. Y. N. Chiu for his useful comments, and Dr. A. Temkin for his interest in this work. 

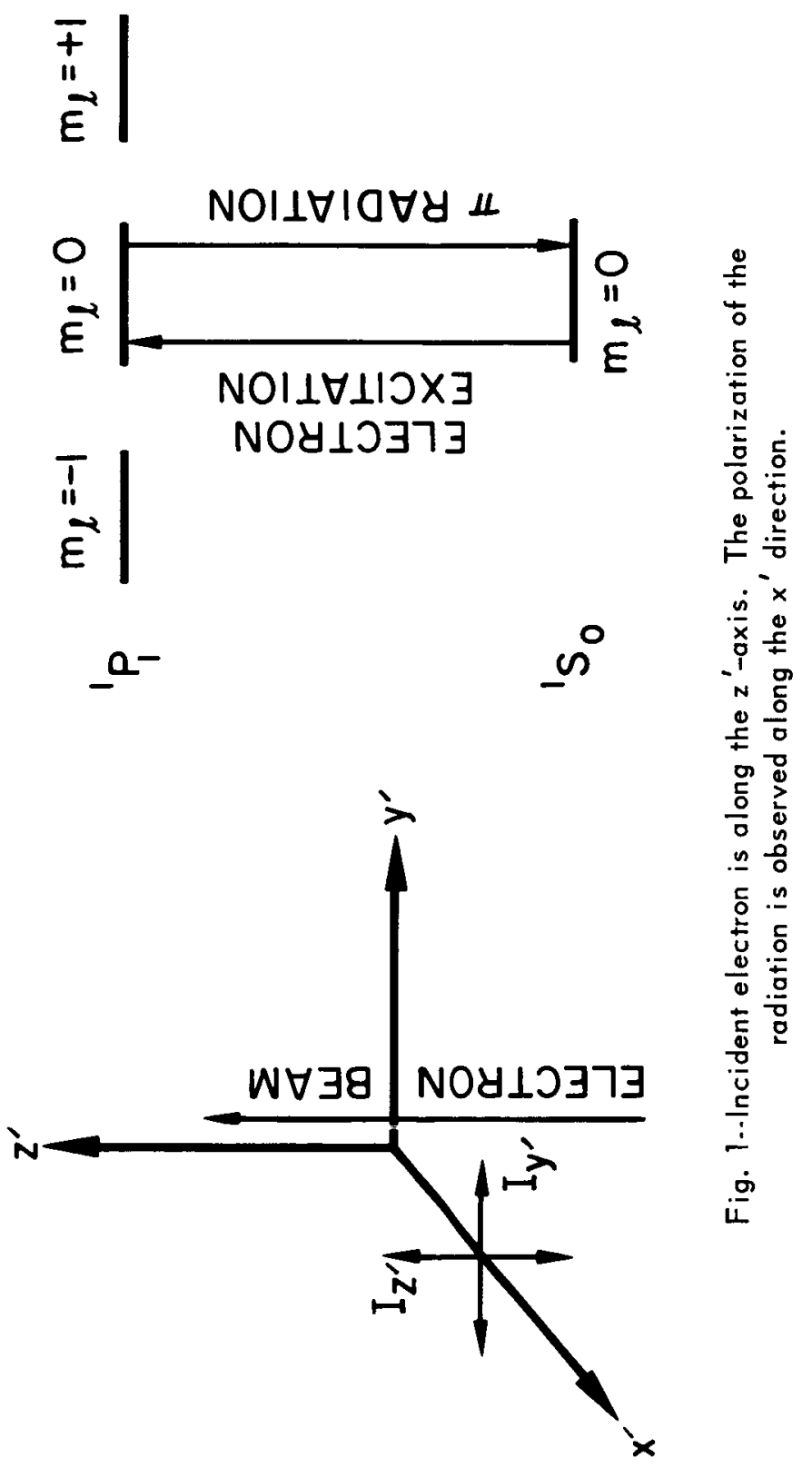

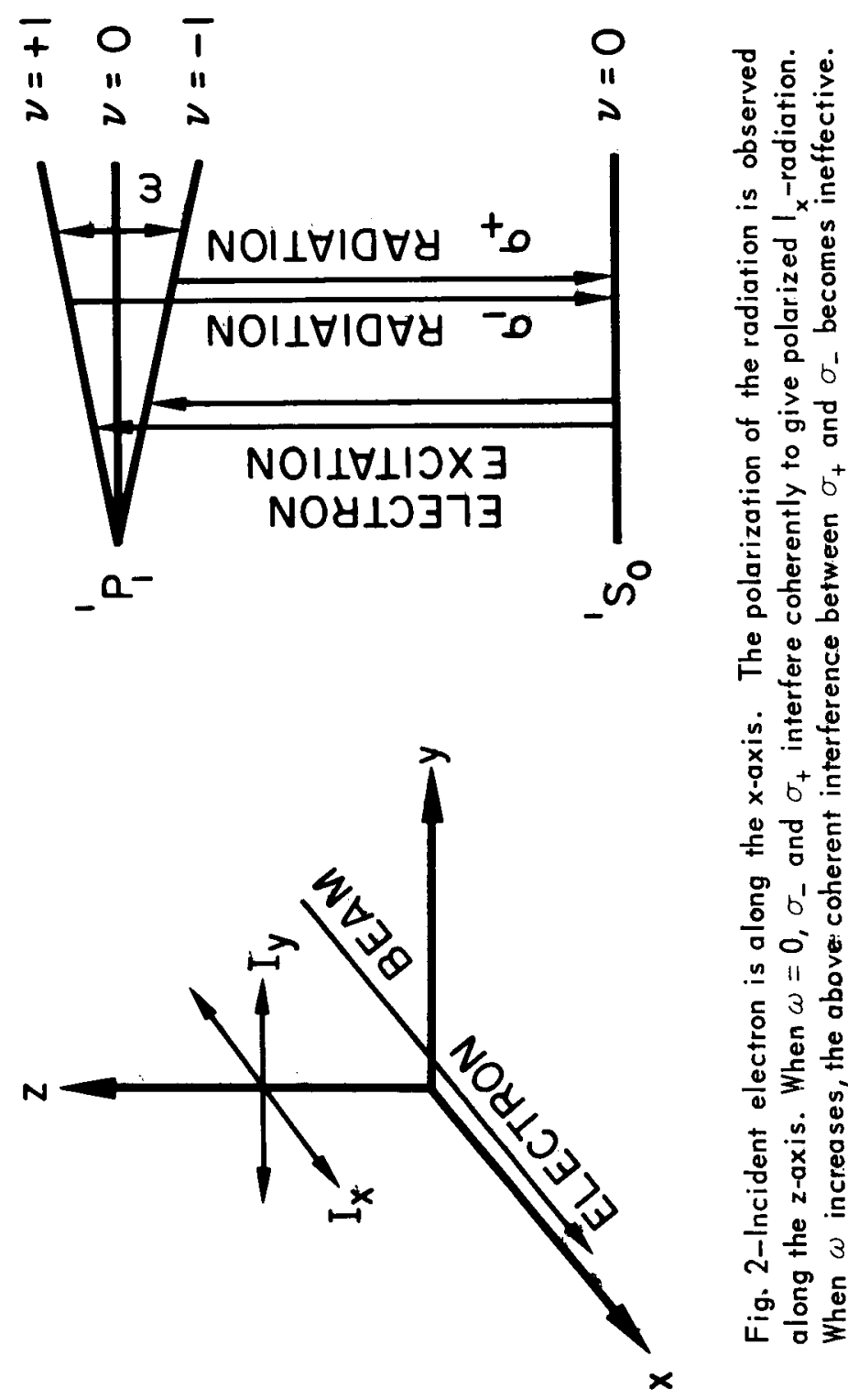

Е

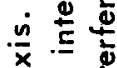

旁 $b^{+}$

号 등

당 $b^{\prime} \frac{1}{0}$

○

. 11 웅

둥

논 동 운

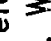

空 $\frac{\dot{n}}{x} \stackrel{0}{0}$

品

드 온

iิ $\frac{0}{6} \frac{5}{0}$ 


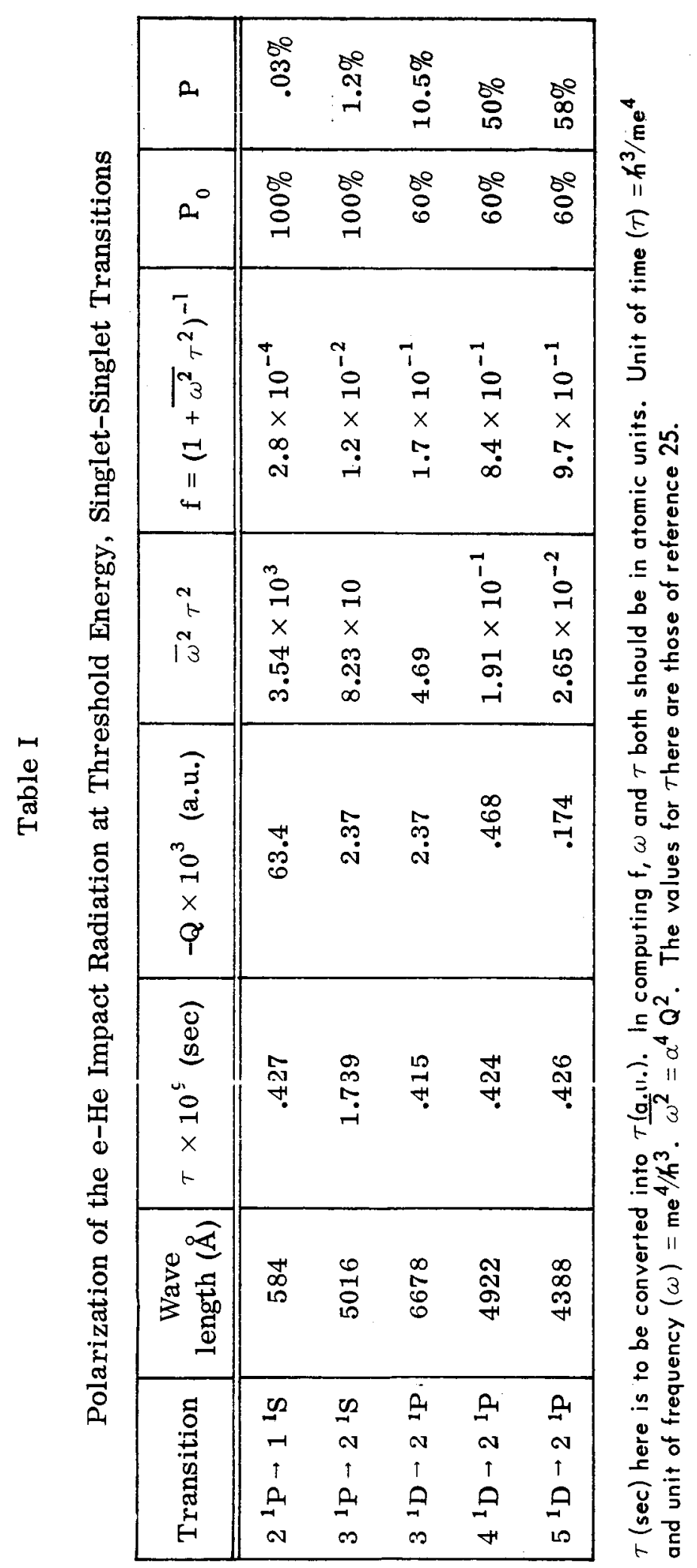




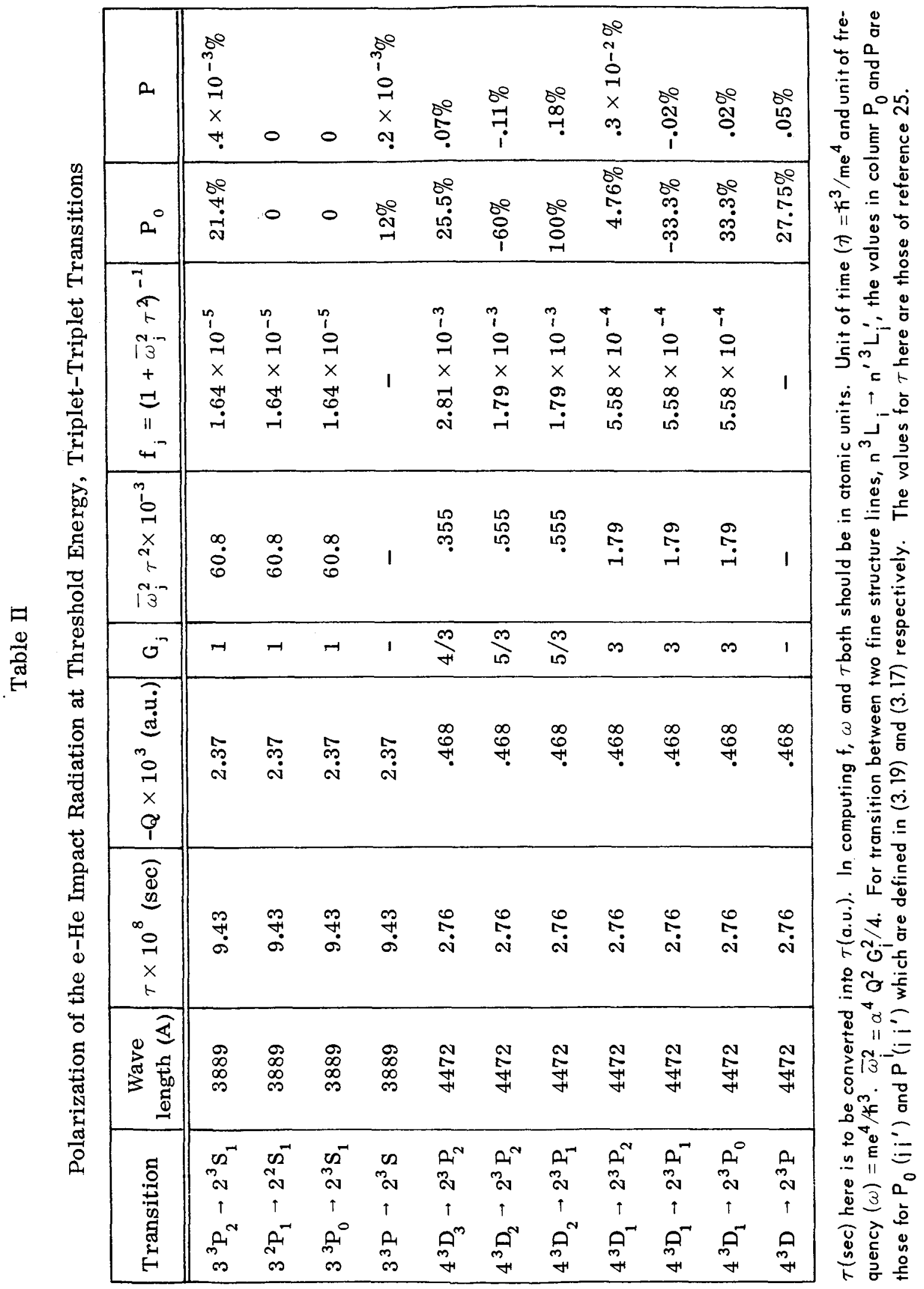




\section{REFERENCES}

1. W. E. Lamb, Phys. Rev. 105, 562 (1957), W. E. Lamb and T. H. Maiman, Phys. Rev. 105, 573 (1957).

2. I. C. Percival and H. J. Seaton, Phil. Trans. Roy. Soc. (London) 113,251 (1958).

3. R. H. McFarland, Phys. Rev. 133, A986 (1964).

4. E. A. Soltysik, A. Y. Fournier and R. L. Gray, Phys. Rev. 153152 (1957).

5. (a) D. W. Heddle and R. G. W. Keesing, in Proceedings of the Fourth International Conference on the Physics of the Electronic and Atomic Collisions, Page 382, Quebec (1965) (Science Bookcrafters, Hastings-on-Hudson, New York, 1965). (b) H. Hafner, H. Kleinpopper, and H. Kruger, p. 386, Op. Cit. Ref. 5a.

6. R. H. McFarland, Phys. Rev. 156, 55 (1967).

7. H. W. B. Skinner, Proc. Roy. Soc. (London) A 112,642 (1926).

8. H. W. B. Skinner and E. T. S. Appleyard, Proc. Roy. Soc. (London) A 117 , 224 (1927).

9. G. Breit, Rev. of Mod. Phys. $\underline{5}, 117$ (1933).

10. P. A. Franken, Phys. Rev. 121, 508 (1961).

11. F. D. Colegrove, P. A. Franken, R. R. Lewis, and R. H. Sands, Phys. Rev. letters $\underline{3}, 420$ (1959). 
12. A. Lurio and R. Novick, Phys. Rev. 134, A608 (1964).

13. W. Hanel, Z. Physik 30, 93 (1924).

14. A. Landman and R. Novick, Phys. Rev. 134, A56 (1964).

15. A. Lurio, R. L. deZafra and R. J. Goshen, Phys. Rev. 134, A1198 (1964).

16. D. K. Anderson, Phys. Rev. 137, A21 (1965).

17. H. Henry Stoke, Physics Today, October 1966, p. 55.

18. $r_{+1}, r_{-1}$ and $r_{0}$ are the components of an unitary spherical vector $r_{m=}$.

19. M. E. Rose, Elementary Theory of Angular Momentum (John Wiley and Sons, Inc., New York, 1961).

20. L. Y. Chow Chiu, Phys. Rev. 137, A385 (1965).

21. Y. N. Chiu, J. Math. Phys. 5,283 (1964).

22. The rotation matrix $D_{m}^{j}{ }_{m}^{j}(0, \beta, 0)$ equals to the reduced matrix $d_{m}^{j}{ }_{m}^{j}(\beta)$ and which is real. Wigner's general expression for $d_{m}^{j}{ }^{j}(\beta)$ can be found in page 52 of reference 19. However, the simple formulae for $j=1$ and 2 can be found in D. M. Brink and G. R. Satchler, Angular Momentum (Clarendon Press, Oxford, 1962), p. 24. The formulae for $\mathrm{j}=3$ has just been listed in Y. N. Chiu, J. Chem. Phys. $\underline{45}, 2985$ (1966).

23. L. C. Biedenharn, J. M. Blatt and M. E. Rose, Rev. Mod Phys. 24, 249 (1952);

A. Simon, J. H. Vanderslui's and L. C. Biedenharm, Oak Ridge National Laboratory Report No. ORNL-1679 (1954).

24. J. C. Slater, Phys. Rev. $\underline{36}, 57$ (1930).

25. L. Goldberg, Ast. Phys. J. $\underline{90}, 414$ (1939). 


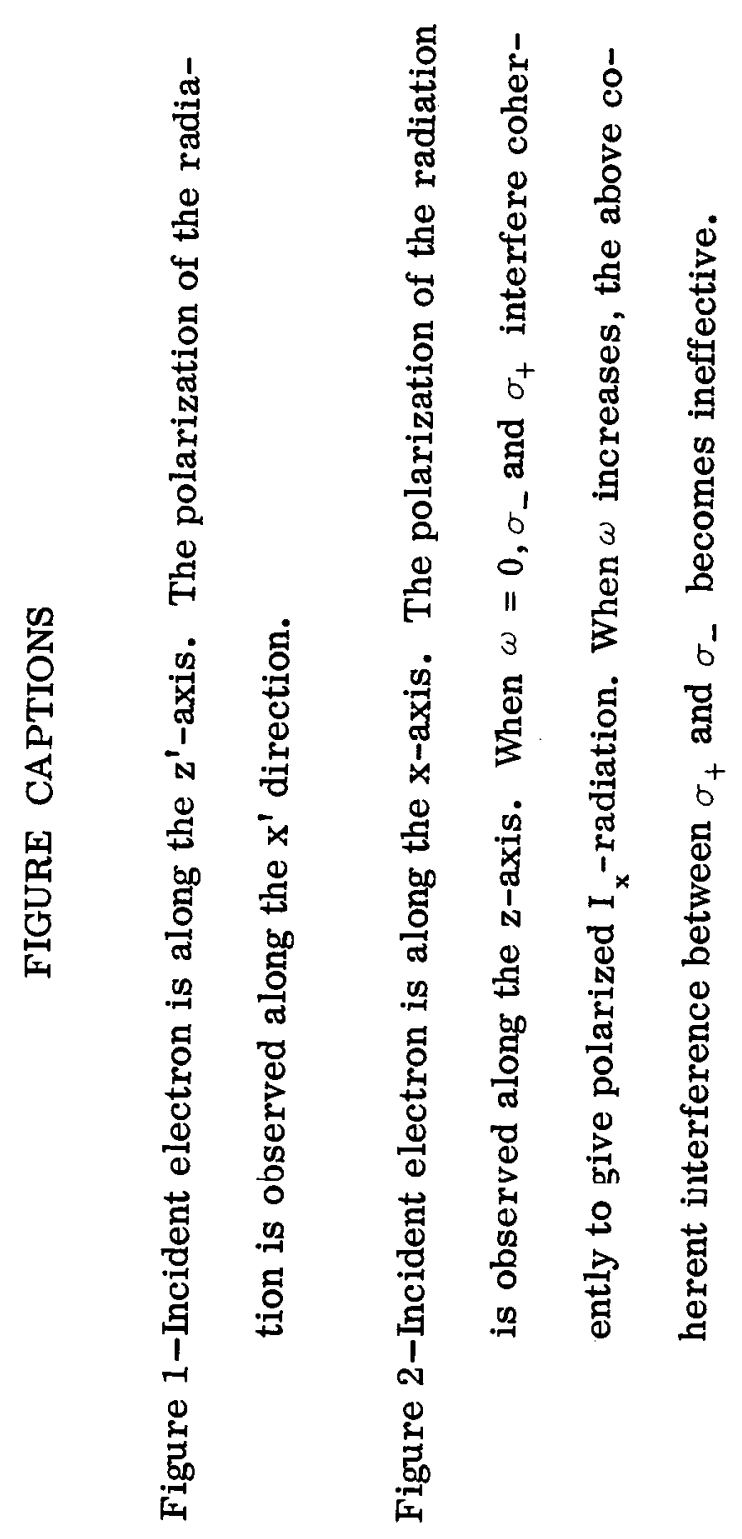

